\title{
HEMODYNAMIC, MORPHOMETRIC AND AUTONOMIC PATTERNS IN HYPERTENSIVE RATS - RENIN- ANGIOTENSIN SYSTEM MODULATION
}

\author{
Fernanda S. Zamo, ${ }^{\mathrm{III}}$ Silvia Lacchini, ${ }^{\mathrm{III}}$ Cristiano Mostarda, ${ }^{\mathrm{I}, \mathrm{II}}$ Silvana \\ Chiavegatto, ${ }^{\text {IV }}$ Ivana C. M. Silva, ${ }^{\mathrm{II}}$ Edilamar Menezes Oliveira, ${ }^{\mathrm{V}}$ Maria Claudia \\ Irigoyen,II
}

doi: $10.1590 / \mathbf{S 1 8 0 7 - 5 9 3 2 2 0 1 0 0 0 0 1 0 0 0 1 3}$

\begin{abstract}
Zamo FS, Lacchini S, Mostarda C, Chiavegatto S, Silva ICM, Oliveira EM, Irigoyen MC. Hemodynamic, morphometric and autonomic patterns in hypertensive rats - renin-angiotensin system modulation. Clinics. 2010;65:(1)85-92.
\end{abstract}

BACKGROUND: Spontaneously hypertensive rats develop left ventricular hypertrophy, increased blood pressure and blood pressure variability, which are important determinants of heart damage, like the activation of renin-angiotensin system.

AIMS: To investigate the effects of the time-course of hypertension over 1) hemodynamic and autonomic patterns (blood pressure; blood pressure variability; heart rate); 2) left ventricular hypertrophy; and 3) local and systemic Renin-angiotensin system of the spontaneously hypertensive rats.

METHODS: Male spontaneously hypertensive rats were randomized into two groups: young ( $\mathrm{n}=13$ ) and adult ( $\mathrm{n}=12$ ). Hemodynamic signals (blood pressure, heart rate), blood pressure variability (BPV) and spectral analysis of the autonomic components of blood pressure were analyzed. LEFT ventricular hypertrophy was measured by the ratio of LV mass to body weight (mg/g), by myocyte diameter $(\mu \mathrm{m})$ and by relative fibrosis area (RFA, \%). ACE and ACE2 activities were measured by fluorometry (UF/min), and plasma renin activity (PRA) was assessed by a radioimmunoassay (ng/mL/h). Cardiac gene expressions of Agt, Ace and Ace2 were quantified by RT-PCR (AU).

RESULTS: The time-course of hypertension in spontaneously hypertensive rats increased BPV and reduced the alpha index in adult spontaneously hypertensive rats. Adult rats showed increases in left ventricular hypertrophy and in RFA. Compared to young spontaneously hypertensive rats, adult spontaneously hypertensive rats had lower cardiac ACE and ACE2 activities, and high levels of PRA. No change was observed in gene expression of Renin-angiotensin system components.

CONCLUSIONS: The observed autonomic dysfunction and modulation of Renin-angiotensin system activity are contributing factors to end-organ damage in hypertension and could be interacting. Our findings suggest that the management of hypertensive disease must start before blood pressure reaches the highest stable levels and the consequent established end-organ damage is reached.

KEYWORDS: SHR; Hypertension; Blood pressure variability; RAS; LVH.

\footnotetext{
I Nephrology Department, Federal University of São Paulo - São Paulo/ SP, Brazil.

II Hypertension Unit, Heart Institute (InCor), Faculdade de Medicina da Universidade de São Paulo - São Paulo/SP, Brazil.

III Department of Anatomy, Institute of Biomedical Sciences, Universidade de São Paulo - São Paulo/SP, Brazil.

Iv Department of Pharmacology, Institute of Biomedical Sciences, Universidade de São Paulo, São Paulo/SP, Brazil.

v Laboratory of Biochemistry, School of Physical Education and Sports, Universidade de São Paulo, São Paulo/SP, Brazil.

Hypertension Unit, Heart Institute (InCor), Faculdade de Medicina da Universidade de São Paulo - São Paulo/SP, Brazil.

Tel.: 55113069.5006

Email: nanda.zamo@terra.com.br

Received for publication on July 24, 2009

Accepted for publication on October 22, 2009
}

\section{INTRODUCTION}

Left ventricular hypertrophy (LVH) and diastolic dysfunction are the early manifestations of cardiac damage in patients with arterial hypertension characterizing hypertensive heart disease. ${ }^{1}$ In response to a variety of mechanical, hemodynamic, hormonal and pathologic stimuli, the heart adapts to increased demands for cardiac work by increasing muscle mass through the compensatory hypertrophic response, which could lead to heart failure. ${ }^{1}$ It is widely recognized that $\mathrm{LVH}$ is a powerful predictor 
of cardiovascular morbidity and mortality, independent from blood pressure (BP) levels and other cardiovascular risk factors. ${ }^{2}$ Indeed, the presence of LVH increases cardiovascular risk in hypertensive patients. ${ }^{3}$

It has been reported that increased BP and blood pressure variability (BPV) are both important determinants of heart damage. ${ }^{4,5}$ In addition, it has been demonstrated that BPV plays a crucial role in the modulation of end organ damage in hypertensive individuals and rats. ${ }^{6-8}$ Indeed, SHR cardiac and vascular hypertrophy have been accompanied by autonomic dysfunction characterized by baroreflex dysfunction, decreased heart rate variability and consequently increased BPV. $4,7,8$

The renin-angiotensin system (RAS) is critical in regulating systemic blood pressure, water and electrolyte balance, and pituitary gland hormones, primarily by the angiotensin II/AT1 receptor subtype system. The importance of the RAS for the pathogenesis of hypertension is underscored by pharmacological interventions. Angiotensin converting-enzyme (ACE) inhibitors and angiotensin receptor antagonists have been used to attenuate or prevent the development of hypertension and its consequences on target organs. ${ }^{9-11}$ In fact, overstimulation of this system can predispose people to cardiovascular disease characterized by excessive vasoconstriction, fibrosis, and cardiac remodeling ${ }^{12}$. Nevertheless, few studies have provided explanations of how RAS is modulated during establishment and maintenance as well as during the time course of the hypertensive process.

This work was undertaken to investigate the effects of the time course of hypertension over 1) hemodynamic and autonomic patterns (blood pressure, blood pressure variability and heart rate); 2) LVH; and 3) local and systemic RAS of the spontaneously hypertensive rat (SHR).

\section{METHODS}

Animals: Male spontaneously hypertensive rats (SHR) were obtained from Central Bioterium of the Federal University of São Paulo, Brazil, at two different ages, four and twelve weeks old, were divided into two groups: young $(n=13)$ and adult $(\mathrm{n}=12)$ rats. The experimental protocol for both groups started eight weeks before the determined ages. In this way, at the end of the protocol, the young group was twelve weeks old and the adult group was twenty weeks old. The twelve-week-old group (young) presented recently established hypertension, while the twenty-week-old group (adult) had suffered the consequences of hypertension for half of their lives, so we could compare different periods of established hypertension. The animals were housed in standard cages (four per cage), and food and water were provided ad libitum.
The environmental temperature was kept at $23+1^{\circ} \mathrm{C}$, and a 12:12 dark-light cycle was maintained throughout the experiment. Every week, animals were weighed, and tail blood pressure was measured. This study was conducted in accordance with the ethical principles in animal research adopted by the Brazilian College of Animal Experimentation (COBEA). This research was approved by the Federal University of Sao Paulo Ethical Committee (\#118/01).

Measurement of hemodynamic signals: Blood pressure (BP) and heart rate (HR) were measured indirectly during the time-course of the experimental protocol, and after eight weeks, they were measured directly. Indirect measurement was performed in both groups using a tail cuff every week. At the end of the study, the rats were anesthetized (ketamine, $90 \mathrm{mg} / \mathrm{kg}$ and xylazine, $10 \mathrm{mg} / \mathrm{kg}$; i.p.) and catheterized in the right femoral artery. Twentyfour hours after catheterization, the signals of the resting BP of conscious rats were recorded $(2 \mathrm{kHz})$ on a beat-to-beat basis using AT/CODAS (DataQ Instruments). A strain-gauge transducer (Statham P23 Db) was used for arterial pressure measurement. The transducer signal was fed to an amplifier (GPA-4, model 2, Stemtech) and then to a 10-bit analog-todigital converter, which interfaced with a computer. The HR was obtained from arterial BP pulses.

Blood Pressure and Pulse Interval Variability: Timedomain analysis consisted of calculating the mean pulse interval (PI) and systolic blood pressure (SBP), as well as their variability as the standard deviation from their respective time series. In the frequency-domain analysis, Fast Fourier Transforming method (FFT) was used to evaluate systolic blood pressure, pulse interval and RR interval variability (SBPV and PIV, respectively). The spectral bands for rats (very low frequency (VLF): 0.0$0.2 \mathrm{~Hz}$; low frequency (LF): 0.2-0.75 Hz; high frequency (HF): $0.8-2.8 \mathrm{~Hz}$ ) were defined according to previous references. ${ }^{13,14}$ Spectral power for LF and HF bands was calculated by means of power spectrum density integration within each frequency bandwidth. The power density of each spectral component was calculated both in absolute values and in normalized units. ${ }^{15}$ Power in LF and in HF for pulse intervals was normalized by calculating the variance minus the power in very low frequency (VLF) and were expressed in normalized units (nu). The sympatho-vagal balance was defined by the LFnu/HFnu ratio. The LF components of the PIV and LF components of the SBPV were considered markers of efferent sympathetic cardiac and vascular modulation, respectively, whereas the HF component of the PIV reflected respiratory-driven vagal modulation to the sinoatrial node. ${ }^{15}$ For frequency-domain analysis, the entire ten-minute time series of blood pressure and pulse, or RR intervals, were evaluated under basal 
conditions using non-parametric methods (FFT), described in detail above. Beat-to-beat values of SBP and PI intervals were used to estimate the cardiac baroreflex sensitivity (BRS) by spectral analysis, using the alpha index for the low-frequency band $(0.20-0.75 \mathrm{~Hz})$. The alpha index analysis evaluates short-term changes in the systolic blood pressure and in the RR interval. This method has been proposed to quantify cause-and-effect events linked with the baroreflex. Indeed, several studies showed a good correlation between the alpha index and the consequences of systemic phenylephrine infusions. ${ }^{16}$

The coherence between the PI and the SBP signal variability was assessed by means of a cross-spectral analysis. The alpha index in the LF band was calculated only when the magnitude of the squared coherence between the PI and SBP signals exceeded 0.5 (range, $0-1$ ). After coherence calculation, the alpha index was obtained from the square root of the ratio between PI and SBP variability in the two major LF bands.

Cardiac hypertrophy: Twenty-four hours after hemodynamic measurements, rats were killed by decapitation, and their hearts were initially perfused with $10 \mathrm{ml}$ of phosphate buffered saline to wash out the blood. After the hearts were stopped at diastole by perfusion with $14 \mathrm{mM} \mathrm{KCl}$, the Left Ventricle (LV; free wall plus septum) was obtained as the tissue remaining after removal of both atria and the free wall of the right ventricle RV. Afterwards, the cardiac chambers were weighed. For determination of cardiac hypertrophy, the LV weight (LVW), RV weight and atria weight were normalized by body weight (BW) to evaluate relative cardiac mass ( $\mathrm{LVW} / \mathrm{BW}$ in $\mathrm{mg} / \mathrm{g}$ ). The LV was submitted to histological evaluation by measuring myocyte diameter and relative fibrosis area. For histological preparation, tissues were immersion-fixed in $4 \%$ formaldehyde and embedded in paraffin, cut into $5 \mu \mathrm{m}$ sections at the level of the papillary muscle and subsequently stained with either hematoxylin-eosin (HE) for cellular structure visualization (to measure myocyte diameter), or with picrosirius (to measure relative fibrosis area). For each evaluation, two randomly selected sections from each LV were visualized by light microscopy at $400 \mathrm{X}$ magnification. Myocytes with visible nuclei and intact cellular membranes were chosen for diameter determination. The widths of individually isolated cardiomyocytes were manually traced across the middle of the nuclei and displayed on a viewing screen with a digitizing pad and determined by a computer-assisted image analysis system (Quantimet 520; Cambridge Instruments, Cambridge, UK). For each animal, approximately 20 visual fields were analyzed, and the observer was blinded to the experimental groups.

Myocardial interstitial relative fibrosis area (RFA) was determined by analyzing the picrosirius-prepared slices through polarized light, as previously reported ${ }^{17}$. In brief, the sections were analyzed using a microscope with $200 \mathrm{X}$ magnification. Twenty fields were selected, and interstitial collagen was determined by a computer-assisted image analysis system (Quantimet 520; Cambridge Instruments), by an observer blinded to the experimental groups. RFA was calculated as a relation of positive collagen area divided by the muscle area in all fields. Perivascular collagen was specifically excluded from this determination.

Serum and Tissue Samples: Blood was collected from the neck wound and centrifuged $\left(1,500 \mathrm{~g}, 15 \mathrm{~min}\right.$ at $\left.4^{\circ} \mathrm{C}\right)$. Blood was collected without an anticoagulant for an ACE activity assay. To determine plasma renin activity and angiotensin II (Ang II) levels, the first $3 \mathrm{~mL}$ of trunk blood were rapidly collected into chilled glass tubes containing a mixture of potassium EDTA $(25 \mathrm{mM}), o$-phenanthrolene $(0.44 \mathrm{mM})$, pepstatin $\mathrm{A}(0.12 \mathrm{mM})$, and 4-chloromercuribenzoic acid (1 $\mathrm{mM})$. This mixture of protease inhibitors prevented the in vitro production and degradation of angiotensin peptides. ${ }^{18}$ The blood was centrifuged, and the plasma or serum were separated and stored at $-20^{\circ} \mathrm{C}$. Hearts were dissected, and atria, right and left ventricles were isolated and weighed. Left ventricles (LV) were stored at $-80^{\circ} \mathrm{C}$ until biochemical and molecular analysis.

Measurement of Ang II: Plasma samples were passed through phenyl silica cartridges (Sep-Pak C18 columns, Waters, Milford, Massachusetts, USA), and the absorbed angiotensin was eluted with methanol. Eluate was dried in a vacuum centrifuge, and the pellet was resuspended in Enzyme ImmunoAssay buffer (EIA buffer), mixed, and centrifuged at 3,000 g for $10 \mathrm{~min}$ at $4^{\circ} \mathrm{C}$. ANG II levels were determined by ELISA, according to the manufacturer's instructions (SPI-BIO). The protein content was determined by the Bradford method (19) by using bovine serum albumin as the standard (Bio-Rad Protein Assay).

Serum and heart ACE and ACE2 activity: $\mathrm{ACE}$ and ACE2 activities were determined by a fluorimetric assay based on the rate of generation of His-Leu by hydrolysis of Hippuryl-His-Leu substrate, as previously reported ${ }^{19}$. Hearts were quickly harvested, rinsed, and homogenized in Tris$\mathrm{HCl}$ buffer, $\mathrm{pH} 7.0$, containing $50 \mathrm{mM} \mathrm{NaCl}$. Homogenates were centrifuged at $1000 \mathrm{~g}$ for $10 \mathrm{~min}$, and the supernatant was frozen at $-20^{\circ} \mathrm{C}$. The protein contents of the samples were measured by Bradford assay using bovine serum albumin as the standard. ${ }^{20}$

Plasma renin activity (PRA) assay: PRA was measured by the angiotensin I radioimmunoassay, using a commercial kit (REN-CT2, CIS Bio International). This assay permits a direct measure of PRA. Results were quantified in a Gama Counter, and the enzyme activity was expressed as $\mathrm{ng} / \mathrm{mL} / \mathrm{h}$ of Ang I. 
Quantification of $m R N A$ levels by real-time polymerase chain reaction $(P C R)$ : Total RNA was isolated from frozen LV samples according to the manufacturer's guidelines. Reverse-transcription and real-time PCR were performed as previously reported. ${ }^{21,22}$ Briefly, total RNA $(2 \mu \mathrm{g})$ samples from the experimental groups were simultaneously reverse-transcribed using a master mix containing oligo(dT) primer and SuperScript II reverse transcriptase (Invitrogen) in a final volume of 201 . cDNA was treated with $2 \mathrm{U}$ of RNase $\mathrm{H}$ (Invitrogen) at $37^{\circ} \mathrm{C}$ for $20 \mathrm{~min}$. Specific primers for the genes Ace (NM_012544), Ace2 (NM_001012006), Agt (NM_134432) and the control gene cyclophilin A (peptidylprolyl isomerase A: Ppia, NM_017101) were designed using the Primer3 software (http://frodo.wi.mit.edu/cgi-bin/primer3/primer3_www. cgi). Forward and reverse primers were positioned in different exons, and specificity was confirmed by a BLAST search (software-assisted search of a nonredundant nucleotide sequence database for rat at http:// www.ncbi.nlm.nih.gov/BLAST). Primers (synthesized by Invitrogen, Sao Paulo, Brazil) had the following sequences: rAceF: 5'-CAGGAACGTGGAACTTGGA-3', rAceR: 5' - CTTTGACGGAAGCATCACC-3', expected product size $=134 \mathrm{bp} ;$ rAce $2 \mathrm{~F}$ : 5'-CGCTGTCACCAGACAAGAA-3', rAce2R: 5'-GCCATTATTTCGTCCAATCC-3', expected product size: 139bp; rAgtF: 5'- TCTACCCTTTTGGGTGCTG-3', rAgtR: 5' -CAAGGAGGATGCTGTTGAGA-3', expected product size: $88 \mathrm{bp}$; and rPpiaF: 5'-AATGCTGGACCAAACACAAA-3', rPpiaR: 5'-CCTTCTTTCACCTTCCCAAA-3', expected product size: $101 \mathrm{bp}$. The relative mRNA expression levels were quantified by an ABI PRISM 7700 Sequence Detection System (SDS) (Applied Biosystems), using the SYBR green methodology. cDNA samples were processed in triplicate for each transcript, and samples without cDNA templates or primers were included as negative controls. The relative expression level of each mRNA in the left ventricle between young and adult rats was calculated according to Vandesompele et al (2002) . ${ }^{23}$ Briefly, the arithmetic mean of the replicated cycling threshold $\left(C_{\mathrm{t}}\right)$ value of each amplicon was transformed to a quantity relative to the sample with the highest expression, taking into account the amplification efficiency of each gene. The raw quantities were subsequently normalized by the reference gene Ppia.

Statistical Analysis: All values are presented as mean \pm standard error. The results were analyzed by a $t$-test. A $\mathrm{p}$ value of less than 0.05 was considered significant.

\section{RESULTS}

\section{Blood pressure, heart rate and blood pressure variabil- ity evaluations}

The indirect BP evaluation performed during the protocol showed an increase in systolic blood pressure (SBP) in the young group (12 weeks old), while the adult group maintained hypertension until 20 weeks of age (figure 1A). The direct hemodynamic evaluation confirmed the increase of resting values of diastolic blood pressure (DBP), systolic blood pressure (SBP), and mean blood pressure (MBP) in adults, when compared to young rats (figure 1B).
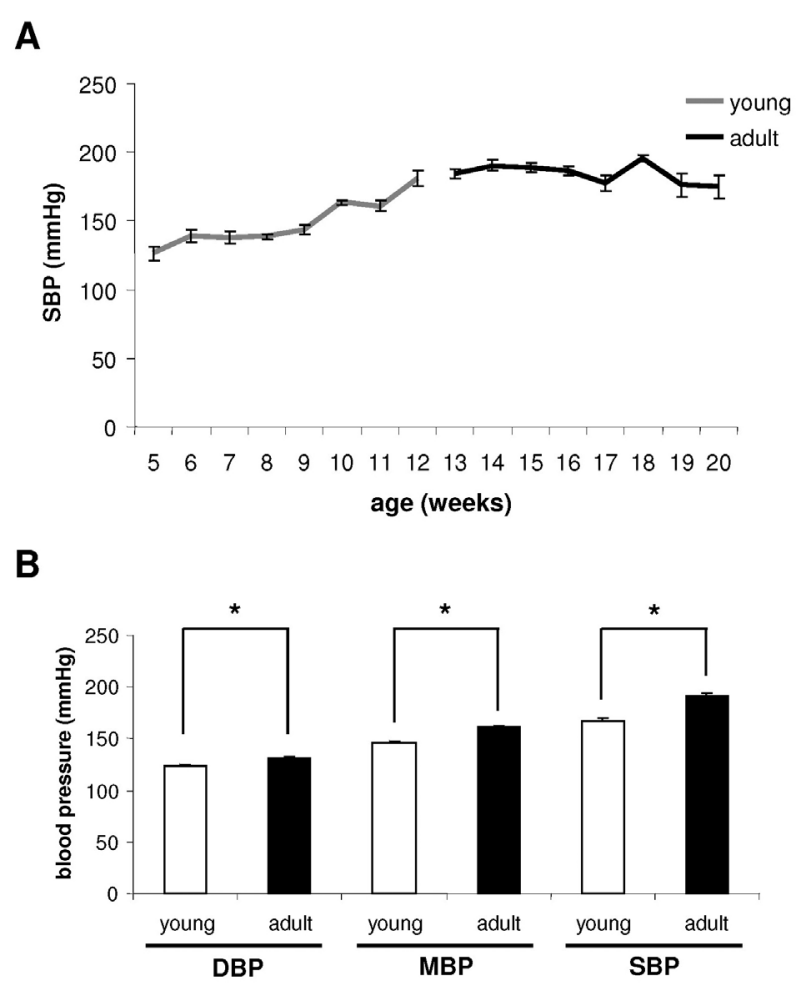

Figure 1 - Indirect and direct blood pressure (BP) measurements. A: tail systolic blood pressure in young and adult rats during experiment. Observe the BP stabilization between the $10^{\text {th }}$ and $12^{\text {th }}$ weeks of age. B: direct systolic $\mathrm{BP}(\mathrm{SBP})$, mean BP (MBP) and diastolic BP (DBP) in young and adult rats. Observe that adult rats presented increases in $\mathrm{BP} . * \mathrm{p} \leq 0.05$

In addition, our data showed increased systolic blood pressure variability in adult rats, which was accompanied by an increased LF component of systolic blood pressure variability when compared to young SHR. Moreover, a marked reduction in the alpha index in the adult rats indicates an age-associated progressive reduction in baroreflex control of circulation in these animals.

Table 1 shows the results obtained by direct measurements. 
Table 1 - Baroreflex sensitivity (alpha index), pulse interval variability (PIV) and systolic blood pressure variability (SBPV) in time and frequency domains (low frequency, LF and high frequency, HF)

\begin{tabular}{lcc}
\hline & Young $(\mathrm{n}=7)$ & Adult $(\mathrm{n}=8)$ \\
\hline PIV $\left(\mathrm{ms}^{2}\right)$ & $78.73 \pm 12.25$ & $65.75 \pm 10.08$ \\
SBPV $\left(\mathrm{mmHg}^{2}\right)$ & $47.93 \pm 8.32$ & $77.96 \pm 9.06 *$ \\
LF of SBPV $\left(\mathrm{mmHg}^{2}\right)$ & $9.49 \pm 1.95$ & $22.34 \pm 3.26 *$ \\
HF of SBPV $\left(\mathrm{mmHg}^{2}\right)$ & $2.61 \pm 0.63$ & $5.64 \pm 1.17 *$ \\
Alpha LF index $(\mathrm{ms} / \mathrm{mmHg})$ & $0.76 \pm 0.1$ & $0.49 \pm 0.07 *$ \\
\hline
\end{tabular}

Data are shown as mean \pm SEM. $* \mathrm{p} \leq 0.05$

\section{Cardiac hypertrophy}

To determine the presence and extent of cardiac hypertrophy, it was necessary to measure body weight. Body weight (BW) increased in young animals and reduced in adults. Young rats started with a weight of $66 \pm 3 \mathrm{~g}$ and finished with a weight of $236 \pm 5 \mathrm{~g}$, while adult rats started at $221 \pm 5 \mathrm{~g}$ and finished at $167 \pm 5 \mathrm{~g}$. It is important to reiterate that body weight was used at the end of the experiment to calculate relative mass of cardiac atria, RV and LV.

The development of heart hypertrophy was investigated by means of the atria weight/BW, RV weight/BW, and $\mathrm{LV}$ weight/BW ratios. The LVW/BW ratio was increased in adult rats when compared to young rats $(2.86 \pm 0.04$ vs. $2.63 \pm 0.05$ $\mathrm{mg} / \mathrm{g}$, respectively, $\mathrm{p}=0.001)$. Atria weight/BW $(0.17 \pm 0.01$ vs. $0.15 \pm 0.01 \mathrm{mg} / \mathrm{g}$, in adult rats) and RV/BW $(0.72 \pm 0.03$ vs. $0.63 \pm 0.03 \mathrm{mg} / \mathrm{g}$, in adult rats) ratios were not different between the groups. Additionally, the adults showed a significant increase in myocyte diameter compared to young rats $(18.2 \pm 0.48$ vs. $12.5 \pm 0.3 \mu \mathrm{m}$, respectively, $\mathrm{p}<0.00001)$.

To evaluate whether cardiac hypertrophy was accompanied by cardiac fibrosis, collagen characteristics were evaluated by the relative fibrosis area. In Table 2, the collagen quantification showed that interstitial RFA was three-fold increased in adult rats when compared to young rats $(1.51 \pm 0.17$ vs. $0.52 \pm 0.05 \%, \mathrm{p}=0.001)$.

\section{Biochemical and molecular analysis of the renin-angio- tensin system}

Renin-angiotensin system component activities were studied in serum, plasma and hearts. As shown in Table 3, both serum Ace activity and angiotensin II levels did not change. Moreover, both heart ACE and ACE2 activities decreased in adult rats, while plasma renin activity increased. On the other hand, angiotensinogen, Ace and Ace2 gene expression in the heart did not change between young and adult rats.
Table 2 - Histological analysis of LV myocyte diameter and relative fibrosis area (RFA)

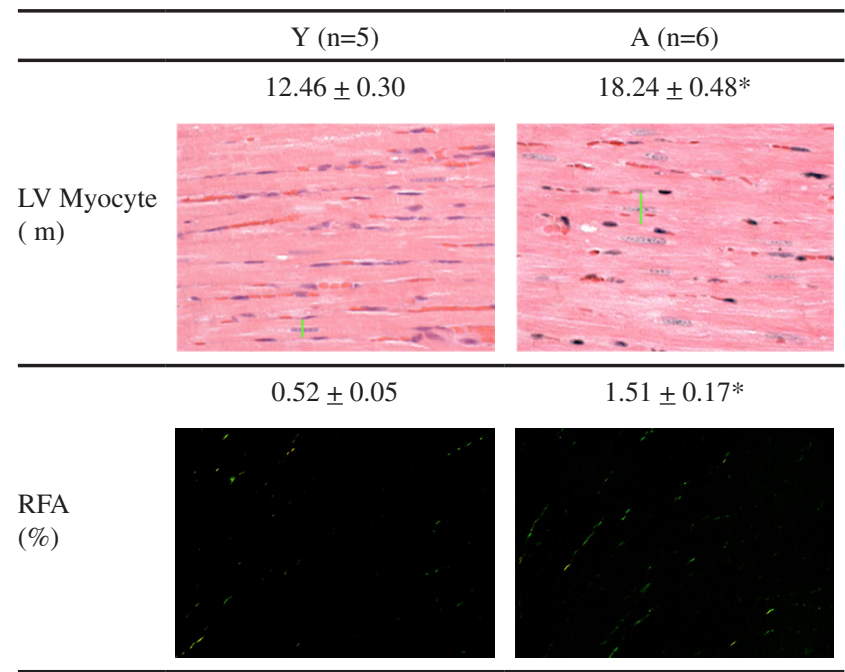

Y: young $(\mathrm{n}=5) ;$ A: adult $(\mathrm{n}=6)$. * $\mathrm{p}<0.001$.

Table 3 - Expression and activity of renin-angiotensin system components

\begin{tabular}{lcc}
\hline & Young $(\mathrm{n}=8)$ & Adult $(\mathrm{n}=8)$ \\
\hline Serum ACE activity $(\mathrm{UF} / \mathrm{min} / \mathrm{mL})$ & $28228 \pm 716$ & $28040 \pm 712$ \\
Heart ACE activity $(\mathrm{UF} / \mathrm{min} / \mathrm{mg})$ & $1579 \pm 108$ & $1010 \pm 86 *$ \\
Heart ACE2 activity $(\mathrm{UF} / \mathrm{min} / \mathrm{mg})$ & $769 \pm 99$ & $622 \pm 76 *$ \\
Plasma renin activity $(\mathrm{ng} / \mathrm{mL} / \mathrm{h})$ & $0.45 \pm 0.07$ & $1.36 \pm 0.05 *$ \\
Angiotensin $\mathrm{II}(\mathrm{pg} / \mathrm{mL})$ & $42.5 \pm 0.77$ & $42.1 \pm 1.39$ \\
Angiotensinogen expression $(\mathrm{AU})$ & $0.771 \pm 0.111$ & $1.103 \pm 0.266$ \\
Heart Ace expression $(\mathrm{AU})$ & $0.945 \pm 0.155$ & $0.965 \pm 0.197$ \\
Heart Ace2 expression $(\mathrm{AU})$ & $0.815 \pm 0.231$ & $0.761 \pm 0.148$ \\
\hline
\end{tabular}

Data are shown as mean \pm SEM. $* \mathrm{p}<0.05$

\section{DISCUSSION}

As expected, blood pressure and systolic blood pressure variability were increased in older SHR (20 weeks old) in comparison with young rats (12 weeks old), indicating progressive activation of hypertensive mechanisms by either sympathetic activity and/or the renin-angiotensin systems in these rats, a fact also documented in humans. ${ }^{24}$ Although previous studies have evaluated BP rhythms in hypertensive rats, information regarding hemodynamic changes during the time-course of hypertension is not very consistent. ${ }^{25}$ This is probably due to the different methods used to measure BP as well as the age of the animals used in each study. El Mas and colleagues (2005), ${ }^{26}$ using telemetry monitoring, observed in 14-27-week-old SHR similar results to those observed in the present study, but they did not analyze any structural alterations. They also did not have any data on the rats before the establishment of stable hypertension. 
Moreover, it is well known that the complications of hypertension, including end-organ damage and microvascular rarefaction, are related not only to an increase in BP levels but also to changes in BP fluctuations around the mean BP values..$^{27,28}$ In the present study, we have observed an increase of $63 \%$ in SBPV in adult SHR when compared to the young SHR group, indicating impairment of the BP control mechanisms from 12 to 20 weeks during the time course of hypertension in SHR. These changes in SBPV were accompanied by the maintenance of hypertension, with values that were kept at the highest levels observed in this experiment. The mechanism of this impairment may be attributed to an increase in sympathetic modulation to the vessels, probably associated with reduced baroreflex sensitivity observed in young SHR and also impaired in adults. In fact, a $100 \%$ enhancement in the LF component of SBPV may account for worsened BP control while no changes were observed in HR variability, (VPI). Similarly, an increase of 50\% in BPV was observed in adult SHR (17-24 weeks old) when compared with WKY controls at the same age, ${ }^{25}$ indicating a role for hypertension in the development of this pathophysiological adjustment.

Interestingly, age-related changes in hypertension as well as changes in the neurogenic mechanisms of BP control are associated with the development of LV cardiac hypertrophy. In fact, not only hypertension but also BPV have been associated with the severity of end-organ damage on a longitudinal basis. ${ }^{29}$ In addition, LV myocyte diameter and collagen quantification were proportionally increased in 20-week-old SHR when compared with the young group, reinforcing the age-related activation of remodeling mechanisms in the heart of these hypertensive animals. In contrast, changes in the atrium and $\mathrm{RV}$ weight were not detected in either observation period in this study, suggesting that it was too early to observe alterations in these heart structures.

The findings regarding activity of the renin-angiotensin system showed that some components are activated in young SHR, at the beginning of hypertension, and this activation is unchanged in adult rats. Recently, Li et al. (2008) ${ }^{30}$ showed that gene expression of RAS components is increased in SHR compared to normotensive Wistar rats. However, the observation of decreased ACE activity in the adult heart may be related to an adaptation to hypertrophy, corroborating the finding of $\mathrm{Li}$ et al. (2008) $)^{30}$ who also showed that Ace gene expression in adult SHR was not different from that of normotensive rats. On the other hand, the decrease of ACE2 activity is also probably related to the heart hypertrophy due to the possible loss of protective mechanisms in hypertensive rats. It is important to consider that ACE and ACE2 tissue activity regulation are occurring in the hypertrophied hearts of hypertensive rats. Furthermore, these decreases in activities are not accompanied by changes in gene expression (as shown in Table 3).
Another important finding is that plasma renin activity is increased in adult rats, suggesting that the RAS activation is due to the well-described neurogenic hypertension in $\mathrm{SHR}^{31}$. This RAS activation may not be essential during the adaptation to hypertension, but it must be essential in a late process, either related to heart failure ${ }^{32}$ or to vascular injury. ${ }^{33}$

The renin-angiotensin system and the autonomic system have a bidirectional relationship, and it is difficult to separate their effects in order to differentiate the sequence of events. Both the sympathetic nervous system and RAS are important blood pressure regulatory factors. ${ }^{34,35}$ Angiotensin receptors and other components of the RAS cascade are widely distributed in the brain ${ }^{36}$ and exert a stimulatory influence on the sympathetic nervous system. Sympathetic hyperactivity is associated with an increase in RAS, while baroreflex sensitivity is influenced by RAS vasoactive peptides. ${ }^{36,37}$ Therefore, the interactions of both systems are responsible for the cardiovascular derangements observed in hypertension. It is known that local RAS plays a key role in the cardiac hypertrophy process. ${ }^{30}$ Additionally, both increased BP and blood pressure variability (BPV) are important factors that stimulate the overactivity of local RAS, although BPV seems to play a larger role..$^{38}$ Considering that sympathetic hyperactivity could be a causal factor of RAS and autonomic dysfunction, we may consider it to maintain the functionality of both dysfunctions.

\section{CONCLUSION}

The findings of the present study demonstrated that BP increased during the observation period of young SHR (from 4 to 12 weeks of age), while it was maintained at an elevated level between the $12^{\text {th }}$ to $20^{\text {th }}$ weeks of age in the adult group. It is important to highlight that these differences were accompanied by increases in SBPV, cardiac hypertrophy and PRA, alongside decreases in baroreflex sensitivity and in heart tissue ACE and ACE2 activities. These observations may indicate that autonomic dysfunction and RAS activity are contributing factors to end-organ damage in hypertension, which have probably influenced additional impairment in autonomic dysfunction by changing vessels and heart structures. All these findings strongly suggest that the management of hypertensive disease must be started before BP reaches its highest stable levels and before resultant end-organ damage is established.

\section{ACKNOWLEDGMENTS}

We thank Fernandes T and Magalhaes FC for assistance with biochemical techniques.

Funding: "Fundação de Amparo à Pesquisa do Estado de São Paulo" (01/01129-0 to FSZ, 01/00009-0 to MCI; SC is a research scholar from CNPq- Brazil). 


\section{REFERENCES}

1. Diamond JA, Phillips RA. Hypertensive heart disease. Hypertens Res. 2005;28: 191-202.

2. Agabiti-Rosei E, Muiesan ML. Left ventricular hypertrophy and heart failure in women. J Hypertens Suppl. 2002;20:S34-8.

3. Ruilope LM, Schmieder RE. Left ventricular hypertrophy and clinical outcomes in hypertensive patients. Am J Hypertens. 2008;21:500-8.

4. Parati G, Ravogli A, Frattola A, Groppelli A, Ulian L, Santucciu C, et al. Blood pressure variability: clinical implications and effects of antihypertensive treatment. J Hypertens Suppl. 1994;12:S35-40.

5. Su DF, Miao CY. Reduction of blood pressure variability: a new strategy for the treatment of hypertension. Trends Pharmacol Sci. 2005;26:38890 .

6. Tatasciore A, Zimarino M, Renda G, Zurro M, Soccio M, Prontera $\mathrm{C}$, et al. Awake blood pressure variability, inflammatory markers and target organ damage in newly diagnosed hypertension. Hypertens Res. 2008;31:2137-46.

7. Xie HH, Shen FM, Zhang XF, Jiang YY, Su DF. Blood pressure variability, baroreflex sensitivity and organ damage in spontaneously hypertensive rats treated with various antihypertensive drugs. Eur J Pharmacol. 2006;543:77-82.

8. Su DF. Treatment of hypertension based on measurement of blood pressure variability: lessons from animal studies. Curr Opin Cardiol. 2006;21:486-91.

9. Freslon JL, Giudicelli JF. Compared myocardial and vascular effects of captopril and dihydralazine during hypertension development in spontaneously hypertensive rats. Br J Pharmacol 1983;80:533-43.

10. Oddie CJ, Dilley RJ, Bobik A. Long-term angiotensin II antagonism in spontaneously hypertensive rats: effects on blood pressure and cardiovascular amplifiers. Clin Exp Pharmacol Physiol. 1992;19:392-5.

11. Wu JN, Berecek KH. Prevention of genetic hypertension by early treatment of spontaneously hypertensive rats with the angiotensin converting enzyme inhibitor captopril. Hypertension. 1993;22:139-46.

12. Wright JW, Mizutani S, Harding JW. Pathways involved in the transition from hypertension to hypertrophy to heart failure. Treatment strategies. Heart Fail Rev. 2008;13:367-75.

13. Ushizima MR, Moreira ED, Costa ET, Castiglioni P, Krieger EM, Di Rienzo M, et al. Effects of sinoaortic denervation on blood pressure, pulse interval, sympathetic nerve activity and diaphragmatic electromyogram variability in conscious rats. IEEE Proceedings of Computers in Cardiology. 2001;485-8.

14. Soares PP, da Nóbrega AC, Ushizima MR, Irigoyen MC. Cholinergic stimulation with pyridostigmine increases heart rate variability and baroreflex sensitivity in rats. Auton Neurosci. 2004;113:24-31.

15. Heart rate variability. Standards of measurement, physiological interpretation, and clinical use. Task Force of the European Society of Cardiology and the North American Society of Pacing and Electrophysiology. Eur Heart J. 1996;17:354-81.

16. Pagani M, Somers V, Furlan R, Dell'Orto S, Conway J, Baselli G, Cerutti $\mathrm{S}$, Sleight $\mathrm{P}$, Malliani A. Changes in autonomic regulation induced by physical training in mild hypertension. Hypertension. 1988;12:600-10.
17. Junqueira LC, Bignolas G, Brentani RR. Picrosirius staining plus polarization microscopy, a specific method for collagen detection in tissue sections. Histochem J 1979;11:447-55.

18. Kohara K, Tabuchi Y, Senanayake P, Brosnihan KB, Ferrario CM. Reassessment of plasma angiotensins measurement: effects of protease inhibitors and sample handling procedures. Peptides. 1991;12:1135-41.

19. Araujo MC, Melo RL, Cesari MH, Juliano MA, Juliano L, Carmona AK. Peptidase specificity characterization of C-and N-terminal catalytic sites of angiotensin I-converting enzyme. Biochemistry. 2000;39:8519-25.

20. Bradford MM. A rapid and sensitive method for the quantitation of microgram quantities of protein utilizing the principle of protein-dye binding. Anal Biochem. 1976;72:248-54.

21. Bibancos T, Jardim DL, Aneas I, Chiavegatto S. Social isolation and expression of serotonergic neurotransmission-related genes in several brain areas of male mice. Genes Brain Behav. 2007;6:529-39.

22. Ambar G, Chiavegatto S. Anabolic-androgenic steroid treatment induces behavioral disinhibition and downregulation of serotonin receptor messenger RNA in the prefrontal cortex and amygdala of male mice. Genes Brain Behav. 2009;8:161-73.

23. Vandesompele J, De Preter K, Pattyn F, Poppe B, Van Roy N, De Paepe A, et al. Accurate normalization of real-time quantitative RT-PCR data by geometric averaging of multiple internal control genes. Genome Biol. 2002;18;3:RESEARCH0034

24. Wichi RB, De Angelis K, Jones L, Irigoyen MC. A brief review of chronic exercise intervention to prevent autonomic nervous system changes during the aging process. Clinics. 2009;64:253-8.

25. Minami N, Imai Y, Munakata M, Sasaki S, Sekino H, Abe K, et al Reversed circadian rhythm of blood pressure in adult spontaneously hypertensive rats. J Hypertens Suppl. 1988;6:S70-3.

26. El-Mas MM, Abdel-Rahman AA. Longitudinal studies on the effect of hypertension on circadian hemodynamic and autonomic rhythms in telemetered rats. Life Sci. 2005;76:901-15.

27. Mancia, G., Parati, G. Ambulatory blood pressure monitoring and organ damage. Hypertension. 2000;36:894-900.

28. Penna GL, Garbero R de F, Neves MF, Oigman W, Bottino DA, Bouskela E. Treatment of essential hypertension does not normalize capillary rarefaction. Clinics. 2008;63:613-8.

29. Frattola A, Parati G, Cuspidi C, Albini F, Mancia G. Prognostic value of 24-hour blood pressure variability. J Hypertens. 1993;11:1133-7.

30. Li L, Yi-Ming W, Li ZZ, Zhao L, Yu YS, Li DJ, et al. Local RAS and inflammatory factors are involved in cardiovascular hypertrophy in spontaneously hypertensive rats. Pharmacol Res. 2008;58:196-201.

31. Stegbauer J, Vonend O, Oberhauser V, Sellin L, Rump LC. Angiotensin II receptor modulation of renal vascular resistance and neurotransmission in young and adult spontaneously hypertensive rats. Kidney Blood Press Res. 2005;28:20-6.

32. Silva GJ, Moreira ED, Pereira AC, Mill JG, Krieger EM, Krieger JE. ACE gene dosage modulates pressure-induced cardiac hypertrophy in mice and men. Physiol Genomics. 2006;27:237-44. 
33. Lacchini S, Heimann AS, Evangelista FS, Cardoso L, Silva GJ, Krieger JE. Cuff-induced vascular intima thickening is influenced by titration of the Ace gene in mice. Physiol Genomics. 2009;37:225-30.

34. Dang A, Zheng D, Wang B, Zhang Y, Zhang P, Xu M, et al. The role of the renin-angiotensin and cardiac sympathetic nervous systems in the development of hypertension and left ventricular hypertrophy in spontaneously hypertensive rats. Hypertens Res. 1999;22:217-21.

35. Paton JF, Wang S, Polson JW, Kasparov S. Signalling across the blood brain barrier by angiotensin II: novel implications for neurogenic hypertension. J Mol Med. 2008;86:705-10.

36. Ganten D, Lang RE, Lehmann E, Unger T. Brain angiotensin: on the way to becoming a well-studied neuropeptide system. Biochem Pharmacol $1984 ; 15 ; 33: 3523-8$
37. Averill DB, Diz DI. Angiotensin peptides and baroreflex control of sympathetic outflow: pathways and mechanisms of the medulla oblongata. Brain Res Bull. 2000;15;51:119-28.

38. Bomtempo CA, Santos GF, Santos RA, Campagnole-Santos MJ. Interaction of bradykinin and angiotensin-(1-7) in the central modulation of the baroreflex control of the heart rate. J Hypertens. 1998;16(12 Pt 1):1797-804.

39. Miao CY, Xie HH, Zhan LS, Su DF. Blood pressure variability is more important than blood pressure level in determination of end-organ damage in rats. J Hypertens. 2006;24:1125-35. 